Binding energies of benzene on coinage metal surfaces: Equal stability on different metals

Friedrich Maaß, Yingda Jiang, Wei Liu, Alexandre Tkatchenko, and Petra Tegeder

Citation: The Journal of Chemical Physics 148, 214703 (2018); doi: 10.1063/1.5030094

View online: https://doi.org/10.1063/1.5030094

View Table of Contents: http://aip.scitation.org/toc/jcp/148/21

Published by the American Institute of Physics

\section{PHYSICS TODAY}

WHITEPAPERS
ADVANCED LIGHT CURE ADHESIVES

READ NOW

Take a closer look at what these environmentally friendly adhesive systems can do
PRESENTED BY

(8) MASTERBOND' 


\title{
Binding energies of benzene on coinage metal surfaces: Equal stability on different metals
}

\author{
Friedrich Maaß, ${ }^{1}$ Yingda Jiang, ${ }^{2}$ Wei Liu, ${ }^{2}$ Alexandre Tkatchenko, ${ }^{3}$ and Petra Tegeder ${ }^{1, a)}$ \\ ${ }^{1}$ Physikalisch-Chemisches Institut, Ruprecht-Karls-Universität Heidelberg, Im Neuenheimer Feld 253, \\ 69120 Heidelberg, Germany \\ ${ }^{2}$ Nano Structural Materials Center, School of Materials Science and Engineering, Nanjing University of Science \\ and Technology, Nanjing 210094, Jiangsu, China \\ ${ }^{3}$ Physics and Materials Science Research Unit, University of Luxembourg, L-1511 Luxembourg, Luxembourg
}

(Received 19 March 2018; accepted 15 May 2018; published online 1 June 2018)

\begin{abstract}
Interfaces between organic molecules and inorganic solids adapt a prominent role in fundamental science, catalysis, molecular sensors, and molecular electronics. The molecular adsorption geometry, which is dictated by the strength of lateral and vertical interactions, determines the electronic structure of the molecule/substrate system. In this study, we investigate the binding properties of benzene on the noble metal surfaces $\mathrm{Au}(111), \operatorname{Ag}(111)$, and $\mathrm{Cu}(111)$, respectively, using temperature-programmed desorption and first-principles calculations that account for non-locality of both electronic exchange and correlation effects. In the monolayer regime, we observed for all three systems a decrease of the binding energy with increasing coverage due to repulsive adsorbate/adsorbate interactions. Although the electronic properties of the noble metal surfaces are rather different, the binding strength of benzene on these surfaces is equal within the experimental error (accuracy of $0.05 \mathrm{eV}$ ), in excellent agreement with our calculations. This points toward the existence of a universal trend for the binding energy of aromatic molecules resulting from a subtle balance between Pauli repulsion and many-body van der Waals attraction. Published by AIP Publishing. https://doi.org/10.1063/1.5030094
\end{abstract}

\section{INTRODUCTION}

Understanding the optoelectronic properties of interfaces between inorganic (metal) surfaces and $\pi$-conjugated organic molecules is fundamental since these interfaces play a key role in molecule-based devices and in their performance. ${ }^{1-4}$ The adsorption configuration, which is governed by the strength of adsorbate/substrate and adsorbate/adsorbate interactions, determines the electronic structure of the molecule/substrate system. Thus, gaining insights into the binding properties such as binding energies of the organic compounds is an important prerequisite. ${ }^{3,5-11}$ Many organic molecules of interest possess a $\pi$-conjugated aromatic electron system. Therefore, pronounced contribution from $\pi$-interactions to their structure and stability when adsorbed on a solid substrate is expected. The simplest aromatic molecule is benzene (Bz); hence, studying the adsorption of $\mathrm{Bz}$ on metal surfaces as a prototype adsorbate/substrate system allows us to obtain information on both metal- $\pi$-interactions and lateral interactions. The adsorption properties of $\mathrm{Bz}$ on coinage metal surfaces, i.e., $\mathrm{Au}(111)$, $\mathrm{Ag}(111)$, and $\mathrm{Cu}(111)$, have been investigated experimentally and theoretically in detail. ${ }^{12-26}$ This includes temperatureprogrammed desorption (TPD) measurements to elucidate the binding strength (the desorption energy, $E_{\text {Des }}$ ). However, the values for $E_{\text {Des }}$ available in the literature are spread over a large range, depending on the method used to analyze the TPD

\footnotetext{
a) Author to whom correspondence should be addressed: tegeder@ uni-heidelberg.de
}

data. In most cases, the Redhead equation has been applied, which assumes that $E_{\text {Des }}$ and the frequency factor (prefactor) are independent from the adsorbate coverage. ${ }^{27}$ In addition, a guess for the prefactor is needed. For instance, for $\mathrm{Bz} / \mathrm{Ag}(111)$, values ranging from 0.43 to $0.8 \mathrm{eV}$ have been reported depending on the value of the proposed prefactor. ${ }^{12,18,28,29}$ Recently, we determined a desorption energy of $0.68 \pm 0.05 \mathrm{eV}$ for $\mathrm{Bz}$ on $\mathrm{Ag}(111)$ from TPD spectra ${ }^{25}$ utilizing the so-called complete analysis. ${ }^{30}$ For this method, no guess for the prefactor has to be made. Note that $E_{\text {Des }}$ for $\mathrm{Bz} / \mathrm{Ag}(111)$ of 0.68 $\pm 0.05 \mathrm{eV}^{25}$ slightly differs from the value elucidated here since we improved our analysis procedure as described in Sec. II.

In this work, we present a detailed analysis of TPD data to elucidate coverage-dependent binding energies of $\mathrm{Bz}$ adsorbed on $\mathrm{Au}(111), \operatorname{Ag}(111)$, and $\mathrm{Cu}(111)$. In addition, first-principles calculations that account for non-locality of electronic exchange and correlation effects have been applied to determine the binding energies. An excellent agreement between the measured and computed binding energies has been obtained. We observed for all studied systems a decrease of the binding energies with increasing coverage, indicating repulsive lateral interactions. Most surprisingly, we found that the binding strength of benzene is equal within the experimental error (accuracy of $0.05 \mathrm{eV}$ ) on all three coinage metal surfaces, even though the electronic properties of the noble metals are quite different. This suggests the existence of a universal trend for the binding energy of aromatic molecules on metal surfaces. 


\section{METHODOLOGY}

\section{A. Experimental methodology}

All experiments were performed under ultrahigh vacuum conditions at a base pressure of $1 \times 10^{-10}$ mbar. The crystals were mounted onto a liquid nitrogen cooled cryostat and together with resistive heating at a temperature range (measured directly at the substrate via a thermocouple) between $100 \mathrm{~K}$ and $800 \mathrm{~K}$ was achievable and precisely controllable. Crystals were prepared by a standard cleaning procedure including $\mathrm{Ar}^{+}$sputtering and subsequent annealing to $750 \mathrm{~K}$. Bz (purchased from Sigma-Aldrich, anhydrous, $99.8 \%$ ) was degassed by several pump-freeze cycles and dosed via a leak valve. The dosing was monitored by a quadrupole mass spectrometer (QMS), allowing precise dosing of particular initial coverages. The sample temperature during deposition was held at $140 \mathrm{~K}$ to avoid water adsorption (for the preparation of multilayer coverages, a substrate temperature of $120 \mathrm{~K}$ was chosen). To record TPD spectra, the samples were heated with a constant heating rate of $\beta=1 \mathrm{~K} / \mathrm{s}$ and the desorbing Bz was monitored with the QMS at the parent molecular ion mass of $m / e=78 \mathrm{amu}\left(\mathrm{C}_{6} \mathrm{H}_{6}^{+}\right)$.

\section{B. Temperature programmed desorption}

We applied TPD to determine the binding energy of $\mathrm{Bz}$ on the (111)-surfaces of the coinage metals $\mathrm{Au}, \mathrm{Ag}$, and $\mathrm{Cu}$. As mentioned above, for the TPD measurements, the Bz-covered metal substrate is heated with a constant heating rate of $\beta=$ $1 \mathrm{~K} / \mathrm{s}$ and the desorbing molecules are detected with a QMS. The desorption rate can be described by the Polanyi-Wigner equation [see Eq. (1)], with the coverage $\theta$, the desorption order $n$, the pre-exponential factor (prefactor) $v$, the desorption or binding energy $E_{\text {Des }}$, the temperature $T$, and the Boltzmann constant $k_{B}$,

$$
\frac{d \theta}{d t}=-v(\theta) \cdot \theta^{n} \cdot \exp \frac{E_{D e s}(\theta)}{k_{B} T} .
$$

A set of TPD curves with varying initial coverages [see Fig. 1(a)] allows us to apply the so-called complete analysis introduced by King et al.,${ }^{30}$ which is based on the PolanyiWigner equation [Eq. (1)]. Besides the HabenschadenKüppers or leading edge analysis, ${ }^{31}$ for this method, a guess for $v$ is not needed. Furthermore, it is the only method which allows analyzing measurements in order to determine the coverage dependency of $v$ and $E_{\text {Des. }}{ }^{32}$ For a constant or given coverage $\theta_{0}$, Eq. (1) can be written in an Arrhenius-type form [see Eq. (2), with $n=1$ ], in which $\ln \frac{d \theta}{d T}$ represents the normalized QMS intensity and $\beta$ the represents applied heating rate,

$$
\ln \frac{d \theta}{d T}=n \cdot \ln \theta_{0}+\ln \frac{v\left(\theta_{0}\right)}{\beta}-\frac{E_{D e s}\left(\theta_{0}\right)}{k_{B} T} .
$$

In each curve of a set of TPD spectra with different initial coverages $\theta_{i}$, a given coverage $\theta_{0}$ is reached at different temperature, i.e., this temperature depends on the initial coverage of a measured TPD spectrum. Therefore each TPD curve delivers one data point in a $\ln \frac{d \theta}{d T}\left[T\left(\theta=\theta_{0}, \theta_{i}\right)\right]$ versus $T^{-1}\left(\theta=\theta_{0}, \theta_{i}\right)$ Arrhenius-like representation [see Fig. 1(c)]. As Eq. (2) shows, the slope yields the desorption energy as a function of coverage $E_{\text {Des }}\left(\theta_{0}\right)$ and the intercept with the y-axis gives $v_{0}\left(\theta_{0}\right)$. For $\theta_{0} \rightarrow 0$, the desorption energy in the limit of single molecules can be elucidated [see Fig. 1(d)]. Note that the coverage dependence of desorption energies gives insights into lateral adsorbate interactions (see below). ${ }^{33}$

In order to describe the TPD data evaluation procedure in more detail, Fig. 1 illustrates the complete analysis for the $\mathrm{Bz} / \mathrm{Au}(111)$. Figure 1(a) shows the TPD data with initial coverages ranging from 0.03 to $1.3 \mathrm{ML}$ (shaded violet).
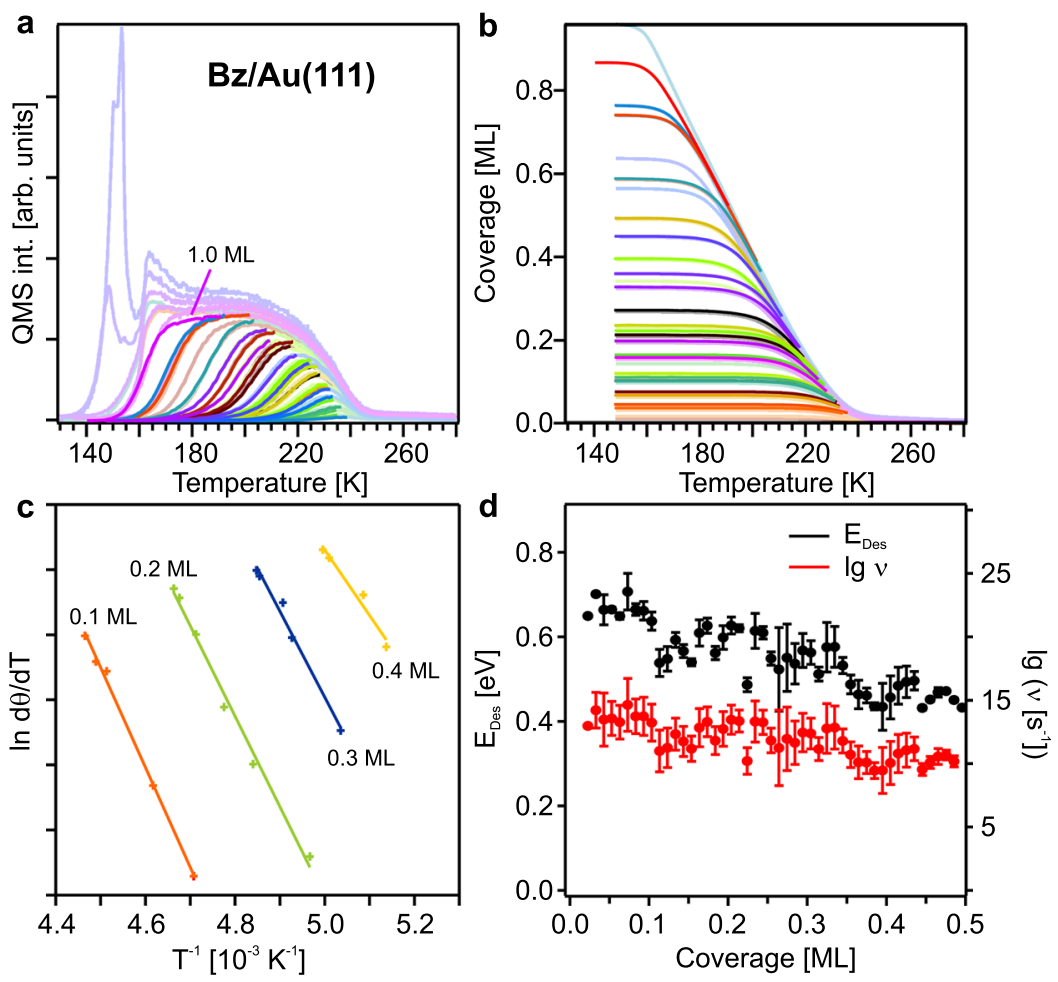

FIG. 1. Description of the complete analysis evaluation exemplarily shown for $\mathrm{Bz}$ on $\mathrm{Au}(111)$. (a) TPD spectra for various initial $\mathrm{Bz}$ coverages. (b) Integrated TPD spectra in which the $y$-axis of the graph corresponds to the given (remaining) surface coverage at the respective temperature. (c) Arrhenius plots for four coverages from the intercept temperatures in (b) and the corresponding QMS intensities at these temperatures in (a). (d) Desorption energies $E_{\text {Des }}$ (black, left axis) and prefactors (red, right axis) as a function of coverage. 
The spectrum with the highest initial coverage and no sign of a shoulder (compressed phase, see below) or a second peak (second/multilayer desorption) was assigned to 1.0 ML, i.e., a complete benzene covered surface. Thus, the area underneath this peak was used for coverage determination. The same definition of $1 \mathrm{ML}$ was applied for $\mathrm{Bz} / \mathrm{Ag}(111)$ and $\mathrm{Bz} / \mathrm{Cu}(111)$ for which we expect no large differences in the absolute coverage. For the analysis, only the data from initial coverages (lowest temperatures) to the highest desorption rates (peak maxima) were used (solid lines) because due to the lateral repulsive interactions (leading to a shift of the desorption peak maximum to lower temperatures with increasing coverage) the falling edges of the TPD spectra lie on each other. Thus, utilization of the falling edges would result in artefact when creating the Arrhenius plots due to experimental inaccuracies.

The complete analysis has successfully been applied for several aromatic molecules on coinage metal surfaces. ${ }^{25,33-35}$ Nevertheless, Nieskens et al. ${ }^{32}$ demonstrated that in the case of repulsive lateral interactions in the adsorbate layer, high quality data with both a high signal-to-noise ratio and temperature resolution (small steps between two measured points) are needed. We addressed these challenges with a series of adjustments like a small sample to QMS distance $(d \approx 10 \mathrm{~mm})$ and a short measurement time for one data point to get small temperature steps $(\Delta T \approx 0.3 \mathrm{~K})$ with the given heating rate $(\beta=1 \mathrm{~K} / \mathrm{s})$.

\section{First-principles calculations}

All density functional theory (DFT) calculations in this work were performed using the "tight" computational settings in the FHI-aims (Fritz Haber Institute $a b$ initio molecular simulations) all-electron code. ${ }^{36,37}$ We employed the recently developed many-body dispersion (MBD) method for van der Waals (vdW) interactions ${ }^{38,39}$ coupled to the PerdewBurke-Ernzerhof (PBE) functional ${ }^{40}$ and to the hybrid HeydScuseria-Ernzerhof (HSE06) functional. ${ }^{41,42}$ We refer to the above approaches as PBE+MBD and HSE+MBD. The MBD method computes the long-range correlation energy through the coupled harmonic oscillator model Hamiltonian and treats dipolar vdW interactions to all orders in perturbation theory.

For comparison, we also employed the PBE and HSE functionals, in conjunction with the DFT+vdW ${ }^{\text {surf }}$ method $^{28}$ that extends pairwise vdW corrections to the modeling of adsorbates on surfaces (referred to as $\mathrm{PBE}+\mathrm{vdW}$ surf and HSE+vdW $\left.{ }^{\text {surf }}\right)$. The DFT+vdW ${ }^{\text {surf }}$ method is based on an effective pairwise atom-atom approximation, which includes electrodynamic screening of $\mathrm{vdW}$ interactions by combining intermolecular vdW interactions ${ }^{43}$ with the Lifshitz-ZarembaKohn theory ${ }^{44,45}$ for the dielectric screening within the metal surface. In our calculations, metal surfaces were modelled by six-layer slabs, and we used a $6 \times 6 \times 1$ Monkhorst-Pack mesh for the sampling of the Brillouin zone of the $(4 \times 4)$ and $(3 \times 3)$ surfaces. For geometry optimizations, the molecule and the two uppermost metal layers were fully relaxed, and the metal atoms of the bottom-most four layers were constrained at their bulk positions. The slabs were separated by $50 \AA$ of vacuum to eliminate the interactions between periodic images. The relativistic effects were treated by using scaled zeroth-order regular approximation (ZORA). ${ }^{46}$

\section{RESULTS}

\section{A. Temperature-programmed desorption}

In the following, we first present and discuss the experimental results on the coverage dependent binding energies for benzene on the three (111)-coinage metals obtained from our TPD measurements and applying the complete analysis. Afterwards we discuss our first-principles calculations. Both theory and experiment will demonstrate that for all three metals the binding energies in the vicinity of a single molecule are very similar.

Figure 2(a) shows a series of TPD spectra with different initial coverages in the submonolayer regime for the desorption of $\mathrm{Bz}$ from the $\mathrm{Au}(111)$ surface. The inset displays TPD spectra with higher initial coverages. Three desorption features labeled as $\alpha_{1}, \alpha_{2}$, and $\alpha_{3}$ are found. $\alpha_{1}$ shows clear zero-order desorption characteristics and does not saturate even for higher coverages (data not shown). Therefore, $\alpha_{1}$ can be assigned to the desorption from the multilayer. We attribute $\alpha_{2}$ to desorption from a more densely packed compressed phase, as reported for $\mathrm{Bz} / \mathrm{Ag}(111)^{18}$ or other aromatic organic molecules on noble metal surfaces. ${ }^{33,34,47-51}$ The sub- to monolayer desorption is represented by $\alpha_{3}$. Note that we defined a monolayer to the desorption spectrum shown in red in the inset. The integral of this spectrum is used as a reference to determine the coverage of all other TPD spectra shown in Fig. 2(a). In the monolayer to sub-monolayer regime [see Fig. 2(a)], the falling edges of $\alpha_{3}$ lie on top of each other, while the peak maximum shifts from $235 \mathrm{~K}$ for an initial coverage of $\theta_{i}=0.01 \mathrm{ML}$ by $45 \mathrm{~K}$ to $190 \mathrm{~K}$ for a coverage of $1 \mathrm{ML}$. This behavior is a clear sign for repulsive lateral adsorbate-adsorbate and substratemediated interactions as reported for aromatic molecules on coinage metal surfaces. ${ }^{13,34,52,53}$

Applying the complete analysis evaluation routine, the binding energies for various given coverages $\theta_{0}$ can be determined from the slopes of Arrhenius plots as exemplary shown for four different coverages in Fig. 1(c). The resulting binding energies as a function of coverage for the $\mathrm{Bz} / \mathrm{Au}(111)$ system are shown in Fig. 2(b). The data are fitted linearly (solid line). Thus, the intercept with the y-axis gives the desorption energy in the limit of vanishing coverage $E_{\text {Des }}(\theta \rightarrow 0)$ $=0.68 \pm 0.03 \mathrm{eV}$. A value of $v(\theta \rightarrow 0)=10^{14.0 \pm 0.3} \mathrm{~s}^{-1}$ for the pre-exponential factor is obtained in the limit of zero coverage [see Fig. 1(d)]. For comparison with theoretical data, this experimental value measured at finite temperatures has to be corrected to $E_{\text {Des }}$ at $0 \mathrm{~K}$ by adding $3 / 2 k_{B} T_{\text {Des }}(\approx 0.03 \mathrm{eV})$ giving a value of $0.71 \pm 0.03 \mathrm{eV}$. The slope of the line is $m=-0.49 \pm 0.04 \mathrm{eV} / \mathrm{ML}$ in the coverage regime up to $0.5 \mathrm{ML}$, which is a measure for the repulsive lateral interactions. For comparison, in the literature, one TPD study on $\mathrm{Bz} / \mathrm{Au}(111)$ is available showing TPD spectra as a function of coverage. ${ }^{15}$ The desorption peaks are in the same temperature range as observed here, but a compressed phase is not seen. Additionally, no detailed analysis of the data has been performed, and an estimation of the binding energy using the Redhead formula ${ }^{27}$ led to a value of $0.64 \mathrm{eV}$ at a coverage of $0.1 \mathrm{ML}$ (with a prefactor of $10^{13} \mathrm{~s}^{-1}$ ). At a coverage of $0.1 \mathrm{ML}$, we obtain a $E_{\text {Des }}$ value of $0.63 \pm 0.03 \mathrm{eV}$ and a prefactor of $10^{13.2 \pm 0.3} \mathrm{~s}^{-1}$ [see Fig. 1(d)]. 

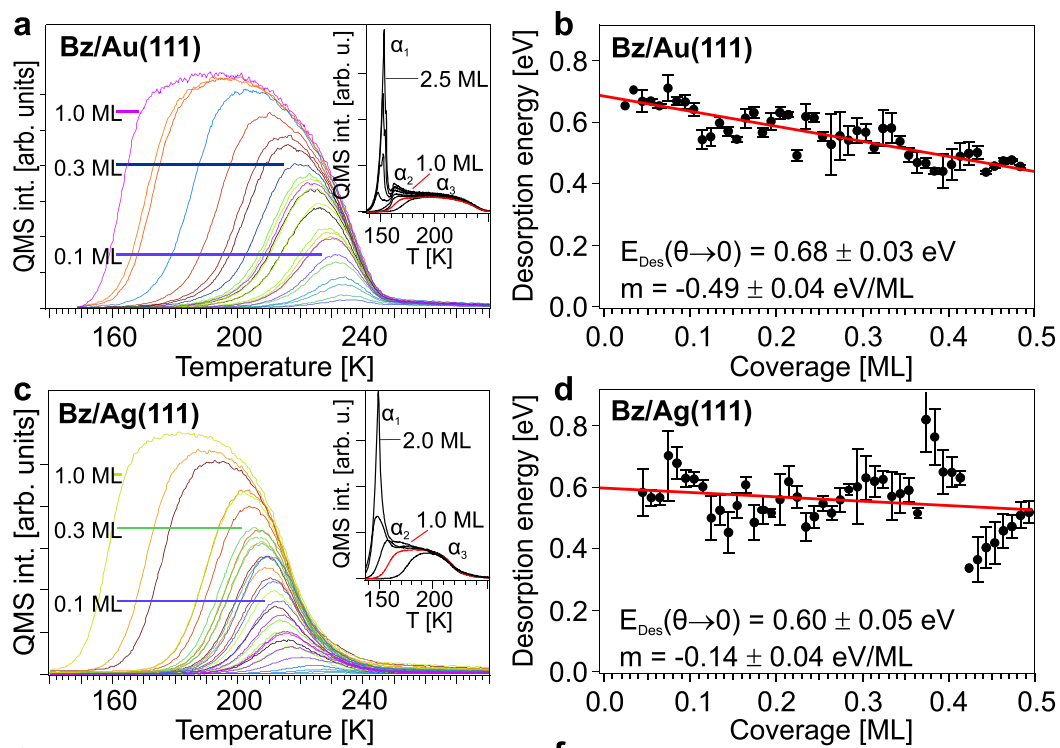

FIG. 2. Coverage dependent TPD spectra of benzene in the low coverage regime on (a) $\mathrm{Au}(111)$, (c) $\mathrm{Ag}(111)$, and (e) $\mathrm{Cu}(111)$. The insets in (a), (c), and (e) show the TPD curve in red, which was assigned to a monolayer coverage as well as the development of a compressed phase $\left(\alpha_{2}\right)$ and the multilayer $\left(\alpha_{1}\right)$ for higher coverages. Desorption energy as a function of Bz coverage on (b) $\mathrm{Au}(111)$, (d) $\mathrm{Ag}(111)$, and (f) $\mathrm{Cu}(111)$.
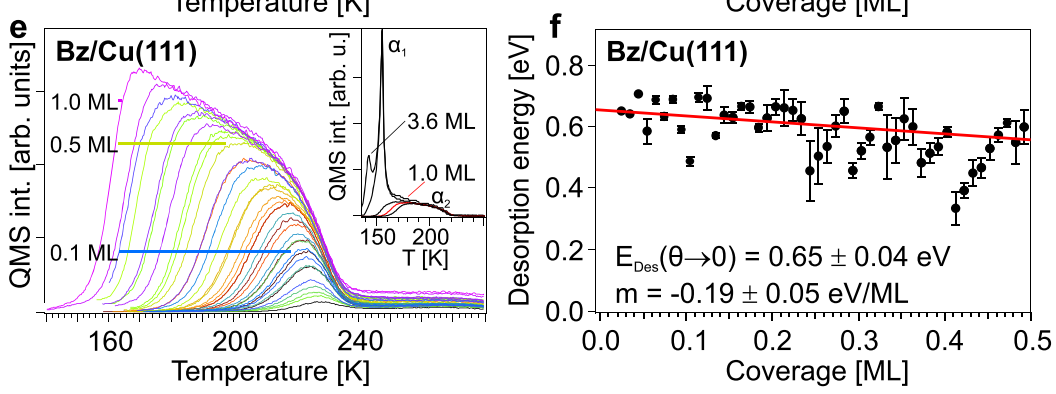

In contrast to the $\mathrm{Bz} / \mathrm{Au}(111)$ system, on $\mathrm{Ag}(111)$ the $\mathrm{Bz}$ compressed phase labeled as $\alpha_{2}$ [see the inset of Fig. 2(c)] is less separated from the multilayer desorption peak $\alpha_{1}$. With increasing coverage, $\alpha_{2}$ seems to merge in $\alpha_{1}$. The analysis of the spectra shown in Fig. 2(c) leads to desorption energies for the given coverages as shown in Fig. 2(d). An extrapolation of the coverage dependent desorption energies results to $E_{\text {Des }}(\theta$ $\rightarrow 0)=0.60 \pm 0.05 \mathrm{eV}$ (corrected to $0 \mathrm{~K}: 0.63 \pm 0.05 \mathrm{eV}$ ). For the pre-exponential factor, we elucidated a value of $v(\theta$

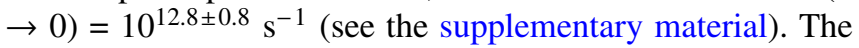
slope of the linear fit is $m=-0.14 \pm 0.04 \mathrm{eV} / \mathrm{ML}$ in the coverage range up to $0.5 \mathrm{ML}$. Hence, the absolute value of the slope is roughly $70 \%$ smaller compared to $\mathrm{Bz} / \mathrm{Au}(111)$, indicating weaker repulsive interactions of $\mathrm{Bz}$ on $\mathrm{Ag}(111)$. Note that for $\mathrm{Bz} / \mathrm{Cu}(111)$ a similar interaction strength is found as for $\mathrm{Bz} / \mathrm{Ag}(111)$ (see below). In the literature, we find a binding energy value extracted from TPD data of $0.57 \mathrm{eV}$ (for $0.1 \mathrm{ML}$ ), which has been determined by using the Redhead expression with a prefactor of $10^{13} \mathrm{~s}^{-1} .18$ At a coverage of $0.1 \mathrm{ML}$, we obtain a value $0.59 \pm 0.05 \mathrm{eV}$ and a prefactor of $10^{12.7 \pm 0.8} \mathrm{~s}^{-1}$ (see the supplementary material).

For Bz on $\mathrm{Cu}(111)$, the TPD spectra are shown in Fig. 2(e) and the corresponding $E_{\text {Des }}$ values as a function of coverage are shown in Fig. 2(f). Note that for $\mathrm{Bz} / \mathrm{Cu}(111)$ the desorption from defect sites, which occurs in the temperature range between 240 and $280 \mathrm{~K}$, is more pronounced than for $\mathrm{Bz} / \mathrm{Ag}(111)$ and $\mathrm{Bz} / \mathrm{Au}(111)$. For $\mathrm{Bz} / \mathrm{Au}(111)$, it is barely seen. For $\mathrm{Bz} / \mathrm{Cu}(111)$, we determined $E_{\text {Des }}(\theta \rightarrow 0)=0.65$ $\pm 0.04 \mathrm{eV}$ (corrected to $0 \mathrm{~K}: 0.68 \pm 0.04 \mathrm{eV}$ ) and for the prefactor $v(\theta \rightarrow 0)$ a value of $10^{13.8 \pm 0.5} \mathrm{~s}^{-1}$ (see the supplementary material), and the linear fit of the coverage-dependent desorption energy results in $m=-0.19 \pm 0.05 \mathrm{eV} / \mathrm{ML}$, thus a similar slope as found for $\mathrm{Bz} / \mathrm{Ag}(111)$. TPD data of $\mathrm{Bz} / \mathrm{Cu}(111)$ have been reported and by using the Redhead formula ${ }^{27}$ a binding energy of $0.59 \mathrm{eV}$ has been suggested at a coverage of 0.1 ML. ${ }^{13,16}$ Based on our measurements, we elucidate a value of $0.63 \pm 0.04 \mathrm{eV}$ for $E_{\text {Des }}$ and a prefactor of $10^{13.5 \pm 0.5} \mathrm{~s}^{-1}$ at a coverage of $0.1 \mathrm{ML}$ (see the supplementary material).

We have to notice that recently Silbaugh et al. ${ }^{54}$ reanalyzed previously published TPD data of $\mathrm{Bz}$ adsorbed on $\mathrm{Au}(111),{ }^{15} \mathrm{Ag}(111),{ }^{12}$ and $\mathrm{Cu}(111),{ }^{13,16}$ respectively, using the Redhead formula and a calculated prefactor of $10^{15.6} \mathrm{~s}^{-1} .55$ This prefactor is one to two orders of magnitude higher than the values we determined on the basis of our experimental results, which are in the range between $10^{14} \mathrm{~s}^{-1}$ and $10^{13} \mathrm{~s}^{-1}$. Additionally, the prefactors are coverage dependent, i.e., they decrease with rising coverage (see the supplementary material).

\section{B. First-principles calculations}

Having obtained precise experimental benchmarks, we now turn to the question whether the state-of-the-art electronic structure calculations are able to achieve quantitative accuracy for Bz on the (111) surfaces of coinage metals. Despite increasing success of DFT, there are still two main problems when applied to adsorption systems: (i) the self-interaction error in the exchange energy, which may result in incorrect electronic energy levels and imperfect electrostatics and (ii) the lack of the long-range $\mathrm{vdW}$ interactions. In this context, here we use a hybrid DFT-HSE exchange-correlation (XC) functional ${ }^{41,42}$ 
to cure a large part of the self-interaction error, which is, for example, visible in the largely improved quadrupole moment of the benzene molecule $\left(6.42 \mathrm{ebohr}^{2}\right.$ in HSE compared to $6.10 \mathrm{ebohr}^{2}$ in PBE; the reference is $6.42 \mathrm{ebohr}^{2}$ at the coupled cluster level of theory). Furthermore, we include many-body vdW correlations with the recently developed DFT+MBD method. ${ }^{38,39}$

For geometry relaxations, we consistently used the $\mathrm{PBE}+\mathrm{vdW} \mathrm{W}^{\text {surf }}$ method. Based on the relaxed geometries, we further employed the MBD method and HSE XC functional for single-point energy calculations. The adsorption energy, $E_{\mathrm{ad}}$, of benzene on metal surfaces has been determined by

$$
E_{\mathrm{ad}}=E_{\mathrm{M}}+E_{\mathrm{Bz}}-E_{\mathrm{Bz} / \mathrm{M}}
$$

where $E_{\mathrm{M}}, E_{\mathrm{Bz}}$, and $E_{\mathrm{Bz} / \mathrm{M}}$ denote the total energy of relaxed bare metal slab, the relaxed gas-phase benzene, and the adsorption system, respectively.

To demonstrate the importance of many-electron $\mathrm{XC}$ effects, we present the adsorption energies for benzene on the (111) surfaces of $\mathrm{Cu}, \mathrm{Ag}$, and $\mathrm{Au}$ in Table $\mathrm{I}$, which allow us to disentangle the contribution of different terms to the adsorption energy. The comparison between PBE+MBD and HSE+MBD calculations demonstrates the crucial reduction of the self-interaction error when applying the HSE XC functional and the ensuing improvement of static electron density and electrostatic interactions. For example, the electrostatic quadrupole moment of the benzene molecule improves from 6.10 ebohr $^{2}$ (with PBE) to 6.42 ebohr $^{2}$ (with HSE), compared to 6.42 ebohr $^{2}$ obtained with a reference coupled cluster wavefunction. $^{25}$

To understand the importance of collective electronic correlation effects in the interaction of benzene with the (111) surface of coinage metals, we proceed to analyze the $\mathrm{HSE}+\mathrm{vdW}$ surf and $\mathrm{HSE}+\mathrm{MBD}$ calculation results. The $\mathrm{vdW}^{\text {surf }}$ method accurately includes the screening of the $\mathrm{vdW}$ interaction inside the extended metal bulk. The MBD method goes significantly beyond the vdW $\mathrm{W}^{\text {surf }}$ and treats the collective correlations within the molecule/solid system to all orders of perturbation theory. ${ }^{38,39}$ Compared to $\mathrm{HSE}+\mathrm{vdW} \mathrm{W}^{\text {surf }}$, the large reduction of binding energies with $\mathrm{HSE}+\mathrm{MBD}$ stems from significant interface polarization redistribution due to many-body correlation effects.
The accurate inclusion of collective non-local XC effects in the HSE+MBD approach leads to excellent agreement with our experimental measurements in both $(4 \times 4)$ and $(3 \times 3)$ unit cells (see Table I). When increasing the coverage, the adsorption energies decrease by $0.02-0.11 \mathrm{eV}$, while the adsorption distances decrease less than $0.04 \AA$. Note that we also performed calculation for the $(5 \times 5)$ unit cell, which essentially yielded the same energies as obtained for the $(4 \times 4)$ unit cell. Thus, we can assume that lateral interactions are converged in our calculations. Therefore we compare our calculated binding energies with the experimental values determined in the limit of vanishing coverage $E_{\text {Des }}(\theta \rightarrow 0)$ for which adsorbate/adsorbate interactions are absent. Generally, a comparison between calculated binding energies for different unit cells and experimental results obtained from TPD has to be done with care since most $\pi$-conjugated aromatic compounds form islands in the sub-monolayer regime due to lateral interactions. For instance, a $(3 \times 3)$ unit cell corresponds to a coverage of $1 / 9 \mathrm{ML}$ and is related to a coverage of $0.62 \mathrm{ML}$ in our experiment ( $1 \mathrm{ML}$ is assigned to the coverage corresponding to the saturation of the $\alpha_{3}$ desorption peaks and $\alpha_{2}$ in the case of $\mathrm{Cu}(111)$; see Fig. 2 and Sec. II). The binding energies for this coverage are included in Table I. In particular, the theoretical and experimental values for $\mathrm{Bz} / \mathrm{Au}(111)$ disagree because for this system the lateral interactions are much stronger compared to $\mathrm{Bz} / \mathrm{Ag}(111)$ and $\mathrm{Bz} / \mathrm{Cu}(111)$. While DFT+vdW calculations are in qualitative agreement with experiment concerning the repulsive nature of lateral interactions, our calculations are still missing the Coulomb-induced effects in the vdW dispersion interactions beyond the dipole approximation. These effects depend on the screening by the underlying surface (hence they will be different for $\mathrm{Cu}, \mathrm{Ag}$, and $\mathrm{Au}$ ) and will lead to an increase of the repulsive interaction as recently demonstrated for intermolecular interactions under confinement. ${ }^{58}$

While the binding energies of benzene on the coinage metal surfaces are similar, interestingly, the adsorption distances differ significantly for the three metals, which suggests that the balance between repulsive and attractive forces is achieved at different distances due to the different vdW radii of the $\mathrm{Cu}, \mathrm{Ag}, \mathrm{Au}$ atoms. However, similar adsorption energies for $\mathrm{Bz}$ on the three surfaces determined in both our

TABLE I. Computed binding energies and adsorption heights for benzene on (111) surfaces of coinage metals at the most preferable sites. All experimental values are corrected to $E_{\text {Des }}$ at $0 \mathrm{~K}$ and for the $4 \times 4$ unit cell the values correspond to the limit of zero coverage (see text). For the $3 \times 3$ unit cell, the corresponding coverage in the experiment is $0.62 \mathrm{ML}$ (see text).

\begin{tabular}{|c|c|c|c|c|c|c|c|c|}
\hline \multirow[b]{2}{*}{ Systems } & \multirow[b]{2}{*}{ Unit cell } & \multicolumn{5}{|c|}{$E_{\mathrm{ad}}(\mathrm{eV})$} & \multicolumn{2}{|c|}{$d(\AA)$} \\
\hline & & PBE+MBD & $\mathrm{HSE}+\mathrm{MBD}$ & PBE+vdW ${ }^{\text {surf }}$ & $\mathrm{HSE}+\mathrm{vdW} \mathrm{W}^{\text {surf }}$ & Expt. & $d_{\mathrm{C}-\mathrm{M}}$ & $d_{\mathrm{H}-\mathrm{M}}$ \\
\hline $\mathrm{Bz} / \mathrm{Cu}(111)$ & $4 \times 4$ & 0.63 & 0.78 & $0.89^{\mathrm{a}}$ & 1.04 & $0.68 \pm 0.04$ & 2.83 & 2.82 \\
\hline $\mathrm{Bz} / \mathrm{Ag}(111)$ & $4 \times 4$ & 0.57 & 0.68 & $0.79^{\mathrm{a}}$ & 0.87 & $0.63 \pm 0.05$ & 2.97 & 2.95 \\
\hline $\mathrm{Bz} / \mathrm{Au}(111)$ & $4 \times 4$ & 0.56 & 0.67 & $0.7^{\mathrm{a}}$ & 0.86 & $0.71 \pm 0.03$ & 3.05 & 3.04 \\
\hline $\mathrm{Bz} / \mathrm{Cu}(111)$ & $3 \times 3$ & 0.52 & 0.67 & $0.79^{b}$ & 0.94 & $0.56 \pm 0.04$ & 2.79 & 2.79 \\
\hline $\mathrm{Bz} / \mathrm{Ag}(111)$ & $3 \times 3$ & 0.52 & 0.60 & $0.73^{\mathrm{b}}$ & 0.81 & $0.54 \pm 0.05$ & 2.96 & 2.95 \\
\hline $\mathrm{Bz} / \mathrm{Au}(111)$ & $3 \times 3$ & 0.51 & 0.62 & $0.73^{b}$ & 0.84 & $0.41 \pm 0.03$ & 3.05 & 3.04 \\
\hline
\end{tabular}

${ }^{\mathrm{a}}$ The binding energy values have been adopted from Ref. 56 .

${ }^{\mathrm{b}}$ The binding energy values have been adopted from Ref. 57 . 
experimental and theoretical study result from a subtle balance between Pauli repulsion and many-body vdW attraction. In other words, for each surface, the adsorption energy reaches its universal value regardless of the precise equilibrium position. We note here that pairwise approaches to the vdW energy coupled with density functionals are unable to correctly reproduce the degeneracy of benzene adsorbed on $\mathrm{Cu}, \mathrm{Ag}$, and $\mathrm{Au} .^{25,56,57}$

\section{CONCLUSION}

In summary, we utilized temperature-programmed desorption (TPD) and first-principles calculations to precisely determine the binding strength of benzene (Bz) on the (111)surface of three coinage metals $\mathrm{Au}, \mathrm{Ag}$, and $\mathrm{Cu}$. The coveragedependent TPD data have been analyzed using the so-called complete analysis. We achieved an accuracy in the binding energy determination of $0.05 \mathrm{eV}$. In the monolayer regime, we observed for all Bz/metal systems a decrease in binding energy with increasing coverage. This effect is assigned to repulsive adsorbate/adsorbate (lateral) interactions. In the limit of zero coverage, the binding strength of $\mathrm{Bz}$ is found to be equal within the experimental error on all three substrates. For Bz/Au(111), the binding energy is $0.68 \pm 0.03 \mathrm{eV}$; for $\mathrm{Bz} / \mathrm{Ag}(111)$, it is $0.60 \pm 0.05 \mathrm{eV}$; and for $\mathrm{Bz} / \mathrm{Cu}(111)$, we obtained a value of $0.65 \pm 0.04 \mathrm{eV}$. The determined prefactors are coverage dependent, i.e., they decrease with increasing coverage. In the limit of a single molecule, they possess values in the range between $10^{14} \mathrm{~s}^{-1}$ and $10^{13} \mathrm{~s}^{-1}$. First-principles calculations that account for non-locality of electronic exchange and correlation effects have been applied to calculate the binding energies. Excellent agreement with the experimental results has been found for the many-body dispersion (MBD) method accounting for van der Waals (vdW) interactions coupled to the hybrid Heyd-Scuseria-Ernzerhof (HSE) functional (HSE+MBD approach). A similar binding strength of $\mathrm{Bz}$ on the three metal surfaces elucidated in both experiment and theory suggests the existence of a universal trend for the binding energy of aromatic molecules on surfaces due to an elaborate balance between Pauli repulsion and many-body vdW attraction. Future first-principles calculations for monolayer coverages should include Coulomb-induced effects in the vdW dispersion energy to determine the relevance of these beyonddipole effects in the lateral interactions between molecules adsorbed on metal surfaces.

\section{SUPPLEMENTARY MATERIAL}

See supplementary material for information about the prefactors as a function of coverage on the coinage metal surfaces.

\section{ACKNOWLEDGMENTS}

F.M. and P.T. acknowledge funding by the Deutsche Forschungsgemeinschaft through the SFB 1249 "NHeteropolycycles as Functional Materials" (Project No. B 06). W.L. acknowledges funding by National Natural Science Foundation of China (Grant No. 51722102).
${ }^{1}$ H. Ishii, K. Sugiyama, E. Ito, and K. Seki, Adv. Mater. 11, 605 (1999).

${ }^{2}$ S. Braun, W. R. Salaneck, and M. Fahlman, Adv. Mater. 21, 1450 (2009).

${ }^{3}$ The Molecule-Metal Interface, edited by N. Koch, N. Ueno, and A. Wee (Wiley-VCH, Weinheim, 2013).

${ }^{4}$ M. Oehzelt, N. Koch, and G. Heimel, Nat. Commun. 5, 4174 (2014).

${ }^{5}$ M. Gruenewald, K. Wachter, M. Meissner, M. Kozlik, R. Forker, and T. Fritz, Org. Electron. 14, 2177 (2013).

${ }^{6}$ R. Forker, M. Gruenewald, and T. Fritz, Annu. Rep. Prog. Chem., Sect. C: Phys. Chem. 108, 34-68 (2012).

${ }^{7}$ S. Bommel, N. Kleppmann, C. Weber, H. Spranger, J. N. P. Schäfer, S. Roth, F. Schreiber, S. H. Klapp, and S. Kowarik, Nat. Commun. 5, 5388 (2014).

${ }^{8}$ H. Yamane, A. Gerlach, S. Duhm, Y. Tanaka, T. Hosokai, Y. Mi, J. Zegenhagen, N. Koch, K. Seki, and F. Schreiber, Phys. Rev. Lett. 105, 046103 (2010).

${ }^{9}$ T. Breuer, M. Klues, and G. Witte, J. Electron Spectrosc. Relat. Phenom. 204, 102 (2015).

${ }^{10}$ M. B. Casu, J. Electron Spectrosc. Relat. Phenom. 204, 39 (2015).

${ }^{11}$ A. F. Jones, B. Chattopadhyay, Y. H. Geerts, and R. Resel, Adv. Funct. Mater. 26, 2233 (2016).

${ }^{12}$ X. L. Zhou, M. E. Castro, and J. M. White, Surf. Sci. 238, 215 (1990).

${ }^{13}$ M. Xi, M. Yang, S. Jo, B. Bent, and P. Stevens, J. Chem. Phys. 101, 9122 (1994).

${ }^{14}$ S. Stranick, M. Kamna, and P. Weiss, Surf. Sci. 338, 41 (1995).

${ }^{15}$ D. Syomin, J. Kim, B. E. Koel, and G. B. Ellison, J. Phys. Chem. B 105, 8387 (2001).

${ }^{16}$ S. Lukas, S. Vollmer, G. Witte, and C. Wöll, J. Chem. Phys. 114, 10123 (2001).

${ }^{17}$ P. Han, B. A. Mantooth, E. C. H. Sykes, Z. J. Donhauser, and P. S. Weiss, J. Am. Chem. Soc. 126, 10787 (2004).

${ }^{18}$ T. J. Rockey, M. Yang, and H.-L. Dai, J. Phys. Chem. B 110, 19973 (2006).

${ }^{19}$ W.-K. Chen, M.-J. Cao, S.-H. Liu, C.-H. Lu, Y. Xu, and J.-Q. Li, Chem. Phys. Lett. 417, 414 (2006).

${ }^{20}$ B. A. Mantooth, E. C. H. Sykes, P. Han, A. M. Moore, Z. J. Donhauser, V. H. Crespi, and P. S. Weiss, J. Phys. Chem. C 111, 6167 (2007).

${ }^{21}$ K. Berland, T. Einstein, and P. Hyldgaard, Phys. Rev. B 80, 155431 (2009).

${ }^{22}$ K. Tonigold and A. Groß, J. Chem. Phys. 132, 224701 (2010).

${ }^{23}$ J. Granatier, P. Lazar, M. Otyepka, and P. Hobza, J. Chem. Theory Comput. 7, 3743 (2011).

${ }^{24}$ W. Reckien, M. Eggers, and T. Bredow, Beilstein J. Org. Chem. 10, 1775 (2014).

${ }^{25}$ W. Liu, F. Maaß, M. Willenbockel, C. Bronner, M. Schulze, S. Soubatch, F. S. Tautz, P. Tegeder, and A. Tkatchenko, Phys. Rev. Lett. 115, 036104 (2015).

${ }^{26}$ J. A. G. Torres, B. Ramberger, H. A. Frühtl, R. Schaub, and G. Kresse, Phys. Rev. Mater. 1, 060803 (2017).

${ }^{27}$ P. A. Redhead, Vacuum 12, 203 (1962).

${ }^{28}$ V. G. Ruiz, W. Liu, E. Zojer, M. Scheffler, and A. Tkatchenko, Phys. Rev. Lett. 108, 146103 (2012).

${ }^{29}$ W. Liu, V. G. Ruiz, G.-X. Zhang, B. Santra, X. Ren, M. Scheffler, and A. Tkatchenko, New J. Phys. 15, 053046 (2013).

${ }^{30}$ D. A. King, Surf. Sci. 47, 384 (1975).

${ }^{31}$ E. Habenschaden and J. Küppers, Surf. Sci. 138, L147 (1984).

${ }^{32}$ D. L. S. Nieskens, A. P. Van Bavel, and J. W. Niemantsverdriet, Surf. Sci. 546, 159 (2003).

${ }^{33}$ M. Schulze, C. Bronner, and P. Tegeder, J. Phys.: Condens. Matter 26, 355004 (2014).

${ }^{34}$ E. R. McNellis, C. Bronner, J. Meyer, M. Weinelt, P. Tegeder, and K. Reuter, Phys. Chem. Chem. Phys. 12, 6404 (2010).

${ }^{35}$ G. Mercurio, E. R. McNellis, I. Martin, S. Hagen, F. Leyssner, S. Soubatch, J. Meyer, M. Wolf, P. Tegeder, F. S. Tautz et al., Phys. Rev. Lett. 104, 036102 (2010).

${ }^{36}$ V. Blum, R. Gehrke, F. Hanke, P. Havu, V. Havu, X. Ren, K. Reuter, and M. Scheffler, Comput. Phys. Commun. 180, 2175 (2009).

${ }^{37}$ V. Havu, V. Blum, P. Havu, and M. Scheffler, J. Comput. Phys. 228, 8367 (2009).

${ }^{38}$ A. Tkatchenko, R. A. DiStasio, Jr., R. Car, and M. Scheffler, Phys. Rev. Lett. 108, 236402 (2012).

${ }^{39}$ A. Ambrosetti, A. M. Reilly, R. A. DiStasio, Jr., and A. Tkatchenko, J. Chem. Phys. 140, 000018 (2014).

${ }^{40}$ J. P. Perdew, K. Burke, and M. Ernzerhof, Phys. Rev. Lett. 77, 3865 (1996).

${ }^{41}$ J. Heyd, G. E. Scuseria, and M. Ernzerhof, J. Chem. Phys. 118, 8207 (2003).

${ }^{42}$ J. Heyd, G. E. Scuseria, and M. Ernzerhof, J. Chem. Phys. 124, 219906 (2006). 
${ }^{43}$ A. Tkatchenko and M. Scheffler, Phys. Rev. Lett. 102, 073005 (2009).

${ }^{44}$ E. M. Lifshitz, Sov. Phys. JETP 2, 73 (1956).

${ }^{45}$ E. Zaremba and W. Kohn, Phys. Rev. B 13, 2270 (1976).

${ }^{46}$ E. van Lenthe, E.-J. Baerends, and J. G. Snijders, J. Chem. Phys. 101, 9783 (1994).

${ }^{47}$ S. Hagen, F. Leyssner, D. Nandi, M. Wolf, and P. Tegeder, Chem. Phys. Lett. 444, 85 (2007).

${ }^{48}$ P. Tegeder, S. Hagen, F. Leyssner, M. Peters, S. Hecht, T. Klamroth, P. Saalfrank, and M. Wolf, Appl. Phys. A 88, 465 (2007).

${ }^{49}$ C. Bronner, B. Priewisch, K. Rück-Braun, and P. Tegeder, J. Phys. Chem. C 117, 27031 (2013).

${ }^{50}$ L. Óvári, Y. Luo, F. Leyssner, R. Haag, M. Wolf, and P. Tegeder, J. Chem. Phys. 133, 044707 (2010).
${ }^{51}$ C. Bronner and P. Tegeder, New J. Phys. 16, 053004 (2014).

${ }^{52}$ G. Mercurio, R. J. Maurer, W. Liu, S. Hagen, F. Leyssner, P. Tegeder, J. Meyer, A. Tkatchenko, S. Soubatch, K. Reuter et al., Phys. Rev. B 88, 035421 (2013).

${ }^{53}$ T. Vondrak and X.-Y. Zhu, J. Phys. Chem. B 103, 3449 (1999).

${ }^{54}$ T. L. Silbaugh and C. T. Campbell, J. Phys. Chem. C 120, 25161 (2016).

${ }^{55}$ C. T. Campbell and J. R. V. Sellers, J. Am. Chem. Soc. 134, 18109 (2012).

${ }^{56}$ Y. Jiang, S. Yang, S. Li, and W. Liu, Sci. Rep. 6, 39529 (2016).

${ }^{57}$ J. Carrasco, W. Liu, A. Michaelides, and A. Tkatchenko, J. Chem. Phys. 140, 084704 (2014).

${ }^{58}$ M. Sadhukhan and A. Tkatchenko, Phys. Rev. Lett. 118, 210402 (2017). 